\title{
COMPOSICIÓN DEL ALUMNADO EN ESCUELAS CHILENAS: UN ANÁLISIS MULTIDIMENSIONAL SOBRE LA DIVERSIDAD DEL SISTEMA ESCOLAR
}

\author{
Cristóbal Villalobos ${ }^{1}$ \\ PAZ RAMÍREZ ${ }^{2}$ \\ ISABEL INFANTE ${ }^{3}$ (iD \\ IGNACIO WYMAN ${ }^{4}$ (iD
}

\begin{abstract}
RESUMEN: La investigación analiza la composición del alumnado de los establecimientos educacionales chilenos desde una perspectiva multidimensional, considerando la dimensión socioeconómica, sociocultural y académica del fenómeno. Utilizando datos secundarios, se categoriza a los establecimientos según el grado de homogeneidad o heterogeneidad en once variables. Adicionalmente, se realiza un Análisis de Correspondencias Múltiples (ACM) que permite analizar la relación entre las distintas variables. Los resultados muestran cuatro perfiles de establecimientos escolares diferenciados. Tres de estos perfiles tienden a tener una composición homogénea con niveles distintos de capital socioeconómico y cultural (alto, medio y bajo), mientras que un cuarto grupo presenta altos niveles de heterogeneidad.
\end{abstract}

Palabras-clave: Composición escolar. Análisis de Correspondencias Múltiples (ACM). Chile. Segregación escolar.

\section{STUDENTS COMPOSITION IN CHILEAN SCHOOLS: A MULTIDIMENSIONAL ANALYSIS ABOUT DIVERSITY IN THE SCHOOL SYSTEM}

\begin{abstract}
This paper analyze the school composition of the Chilean educational system from a multidimensional perspective, taking into account the socioeconomic, sociocultural, and academic dimensions. Using secondary data, schools were categorized according to the degree of homogeneity or heterogeneity in eleven variables. In addition, a Multiple Correspondence Analysis (MCA) was performed to analyze the relationship between the different
\end{abstract}

\footnotetext{
Este artículo se desarrolló en el marco de los proyectos Fondecyt Regular №1150261 “Agrupamiento de estudiantes al interior de la escuela y prácticas educativas. Dispositivos institucionales y pedagógicos desarrollados por establecimientos con altos grados de eficacia escolar en el cierre de brechas de aprendizaje" y Fondecyt Iniciación № 11190198 "Estudiando el funcionamiento, organización y dinámicas del campo educativo. Un análisis de la trayectoria, características, relaciones e influencias en el Chile post-dictadura (1990-2020)”, ambos financiado por la Agencia Nacional de Investigación y Desarrollo de Chile, ANID.

1.Pontificia Universidad Católica de Chile - Centro de Estudios de Políticas y Prácticas en Educación - Santiago, Chile. Email: clvillal@uc.cl 2.Pontificia Universidad Católica de Chile - Santiago, Chile.Email: pazramirez@uc.cl

3.Pontificia Universidad Católica de Chile - Santiago, Chile. Email: iinfante1@uc.cl

4.Pontificia Universidad Católica de Chile - Centro de Estudios de Políticas y Prácticas en Educación - Santiago, Chile.

Email: iawyman@uc.cl.
} 
variables. The results show four profiles of different school types. Three of them are homogeneous with different levels of socioeconomic and cultural capital (low, middle and high), whilst a fourth group of schools presents high levels of heterogeneity.

Keywords: School Composition. Multiple Correspondence Analysis (MCA). Chile. School Segregation.

\section{COMPOSIÇÃO DOS ESTUDANTES EM ESCOLAS CHILENAS: UMA ANÁLISE MULTIDIMENSIONAL SOBRE A DIVERSIDADE NO SISTEMA ESCOLAR}

RESUMO: Este artigo analisa a composição das escolas do sistema educacional chileno a partir de uma perspectiva multidimensional, considerando as dimensões socioeconômica, sociocultural e acadêmica. Por meio do uso de dados secundários, escolas foram categorizadas de acordo com o grau de homogeneidade e heterogeneidade em onze variáveis. Além disso, uma Análise de Correspondências Múltiplas (ACM) foi realizada, a fim de analisar a relação entre as diferentes variáveis. Os resultados mostram quatro perfis diferentes de escolas. Três deles tendem a ter uma composição homogênea, com níveis diferentes de capital socioeconômico e cultural (baixo, médio e alto), enquanto um quarto grupo de escolas apresenta alto nível de heterogeneidade.

Palavras-chave: Composição escolar. Análise de Correspondências Múltiplas (ACM). Chile. Segregação escolar.

\section{Introducción}

$\mathrm{E}$

1 sistema escolar chileno se ha visto enfrentado a sucesivos procesos de transformación de la composición escolar de su alumnado. Durante parte importante del siglo XX, el principal cambio estuvo relacionado con un aumento de la diversidad socioeconómica y cultural del estudiantado, principalmente producto de las masivas campañas de escolarización de las clases bajas y medias-bajas (SERRANO et al., 2012) y la progresiva integración de distintos pueblos originarios al sistema educativo (WILLIAMSON, 2012). Durante las últimas décadas, destaca el crecimiento de la inmigración escolar (RIEDEMANN, 2017), el aumento de cobertura de estudiantes con necesidades educativas especiales (DUK; LOREN, 2010) y la profundización de las brechas socioeconómicas entre establecimientos (BELLEI, 2013), en un contexto marcado por la mantención (y en algunos aspectos, consolidación) de una política orientada por la competencia y las lógicas del mercado educativo (VILLALOBOS, 2016).

En parte como una forma de enfrentar estas transformaciones y sus efectos negativos sobre la equidad y diversidad educativa, durante la última década se han desarrollado una serie de reformas estructurales en el sistema escolar que han buscado disminuir el peso del mercado y aumentar la regulación del sistema (VERGER et al., 2016), promover mayores niveles de mixtura social (BELLEI, 2016) y mejorar la calidad de los docentes y de la educación pública (VALENZUELA; MONTECINOS, 2017). Aunque la evidencia sobre los efectos de estas políticas es aún incipiente, es claro que estas reformas han transformado de manera importante la complejidad del sistema escolar chileno.

Por lo anterior, durante los últimos años, la investigación académica ha desarrollado como uno de sus tópicos más comunes el análisis de la composición de los estudiantes del sistema escolar. 
En este ámbito, el análisis de la composición socioeconómica de los estudiantes ha revelado el alto nivel de estratificación y segregación del sistema escolar chileno respecto del origen social (MIZALA; TORCHE, 2012; VALENZUELA et al., 2014). Adicionalmente, pero de manera incipiente, se han desarrollado estudios focalizados en la composición étnica (ELACQUA, 2012; TREVIÑO et al., 2017), de género (VILLALOBOS et al., 2016), nacionalidad (JOIKO; VÁSQUEZ, 2016) y segregación académica al interior de los establecimientos (TREVIÑO et al., 2016; TREVIÑO et al., 2018). Aunque este conjunto de estudios ha dado importantes luces respecto de los procesos de composición escolar, la evidencia se ha focalizado en entender solo un factor para el análisis de estos fenómenos, sin considerar mayormente las interacciones y conexiones existentes entre las distintas variables de la composición escolar.

Como una forma de llenar este vacío, el presente artículo busca analizar la composición de los estudiantes en los establecimientos educacionales chilenos desde una perspectiva multidimensional. Así, y partiendo de la base de que la composición de los establecimientos puede desarrollar patrones diferenciados de comportamiento, se conceptualizan tres dimensiones centrales de la composición escolar: i) la dimensión socioeconómica; ii) la dimensión cultural; y iii) la dimensión académica. Cada una de estas dimensiones, en su conjunto, busca generar una clasificación según el nivel de homogeneidad y heterogeneidad de los establecimientos educativos. Asimismo, se analizan las posibles diferencias en las tipologías de composición escolar según variables relevantes de los establecimientos, como dependencia, nivel de enseñanza, tamaño de escuela y zona geográfica.

Para desarrollar este objetivo, el artículo se organiza en tres apartados, además de esta introducción. El segundo apartado presenta los principales antecedentes conceptuales de la investigación, profundizando en el concepto de composición escolar, sus principales dimensiones y variables. El tercer apartado detalla la metodología de la investigación, dando cuenta de los datos utilizados y métodos de análisis. El cuarto apartado describe los principales resultados de investigación. Las conclusiones y desafíos próximos en materia de investigación y políticas públicas son parte del último apartado.

\section{Antecedentes conceptuales}

\section{Composición escolar: definiciones e implicancias}

Históricamente, la escuela moderna puede ser considerada una institución clave en la socialización del ser humano. Así, Durkheim (1975) señalaba que una de las funciones esenciales de los sistemas escolares era formar al estudiante como un ser social, insertándolo en un ambiente marcado por roles y funciones, bastante diferenciado del espacio familiar e íntimo. Evidentemente, el sentido, alcance y potencia de esta función estará mediado por las características de los actores que se encuentran en el establecimiento, especialmente en los estudiantes, con los que los niños, niñas y adolescentes comparten la mayor cantidad del tiempo escolar. De ahí la importancia de realizar investigaciones focalizadas en la composición escolar, definido como las distintas características agregadas de los estudiantes que componen un establecimiento educacional, respecto a alguna variable

relevante, como el estatus socioeconómico, el capital cultural, la raza, el género, el rendimiento académico etc. (DUPRIEZ, 2010).

En general, la literatura ha reconocido múltiples aspectos de influencia de la composición escolar. Por una parte, diversas investigaciones han mostrado que ser parte de una escuela donde existe 
una composición escolar integrada entrega beneficios al conjunto de los estudiantes en términos de sus actitudes hacia la diversidad, como la menor disposición a estereotipos y prejuicios (MCDONNELL et al., 2000) y el desarrollo de mayores niveles de generosidad, cooperación y amistad (MOODY, 2001). En esta misma línea, estudios empíricos han revelado que, aunque de manera menos clara y lineal, la existencia de escuelas más heterogéneas también contribuye a potenciar mayores actitudes cívicas, especialmente en lo relacionado con la tolerancia a las minorías, conocimiento y participación cívica y apoyo a la democracia (JANMAAT, 2014; SHAFIQ; MYERS, 2014).

Otro aspecto en el que ha incidido la composición escolar está relacionado con las relaciones que se desarrollan al interior de la escuela. Así, estudios muestran que las características del estudiantado tienen un efecto indirecto sobre el clima escolar, las relaciones entre estudiantes y los niveles de violencia escolar (ASCORRA et al., 2016); y las expectativas y disposición con la que profesores y equipos directivos encaran al grupo estudiantil (KELLY, 2007). De esta manera, la composición escolar también incide en la forma en cómo se desarrolla la vida escolar.

Finalmente, la composición escolar es una variable crítica en distintos indicadores educativos. Por una parte, Freeman y Steidl (2016) han indicado que la homogenización puede afectar variables como la expulsión, la repetición y la disciplina en la escuela. Adicionalmente, existe una gran literatura que ha buscado estimar el efecto académico producto de la interacción de estudiantes con distintas capacidades o habilidades académicas. Aunque es un tema de debate, distintos investigadores han indicado que el grupo de pares ejerce una influencia en los logros académicos de los alumnos (OPDENAKKER; VAN DAMME, 2001), lo que se traduciría en que el hecho de asistir a clases con estudiantes de altas competencias y/o habilidades contribuye a mejoras en el logro académico de los alumnos, especialmente de aquellos con bajos niveles de desempeño (CALDAS; BANKSTON, 1997).

\section{La multidimensionalidad de la composición escolar}

La idea de que los sujetos son agentes multidimensionales es parte importante de las propuestas teóricas del siglo XX, desde la Escuela de Birmingham (HALL, 1980) hasta la teoría de los capitales de Bourdieu (1986). Aunque con distintos argumentos y especificaciones, en todos estos casos se ha puesto énfasis en la idea de que existe una interacción de las distintas categorizaciones o clasificaciones sociales de los sujetos. Esto implica, como indica Costa (2012), reconocer que las condiciones como la nacionalidad, la etnia, el género, la posición intelectual o la clase de las personas se interseccionan y condicionan mutuamente en el espacio/tiempo social, configurando así una multiplicidad que determinan e influencian la posición y disposición de los sujetos.

En el espacio escolar, esto implica un entendimiento multidimensional del problema de la composición escolar. Como indica Dupriez (2010) la composición escolar refiere a la composición y distribución de estudiantes en un establecimiento educativo en distintos aspectos. En nuestro caso, distinguiremos especialmente tres dimensiones de la composición escolar: i) la dimensión socioeconómica; ii) la dimensión cultural; y iii) la dimensión académica.

En primer término, la dimensión socioeconómica refiere a las características económicas y de capital cultural que poseen las familias de los estudiantes que se encuentran en los establecimientos educativos. En general, las variables socioeconómicas refieren al origen social de los estudiantes (clase, status, ocupación y/o ingreso de los padres u otros familiares), a la posesión de ciertos bienes materiales (bienes de consumo, acceso a tecnologías o medios de información) o de otras formas de capital, como 
el capital cultural, político o social (BOURDIEU, 1986). Al respecto, la investigación internacional ha mostrado que el nivel socioeconómico del estudiante sigue siendo un aspecto central para explicar resultados académicos (HANUSHEK; WOESSMAN, 2010), socioemocionales (BRADLEY; CORWYN, 2002) o las cualidades cívicas (JANMAAT, 2013) de los estudiantes. En Chile, se ha estudiado que existe un efecto positivo de composición socioeconómica diversa en el rendimiento académico de alumnos (MIZALA; TORCHE, 2012) y la convivencia escolar (ASCORRA et al., 2016).

En segundo lugar, es posible reconocer una dimensión que puede definirse como sociocultural en la composición escolar. De esta manera, se reconoce que ciertas características culturales generan diferencias relevantes que afectan de manera directa la posición y las oportunidades de los sujetos en el sistema escolar. Aspectos como la nacionalidad, la condición étnica, la raza, el género o la condición de discapacidad del estudiante se convierten en categorías cargadas que impactan en la experiencia educativa. En Chile, se ha mostrado que la composición de género mixta (es decir, donde existen estudiantes hombres y mujeres) es, en general, favorable en aspectos socioemocionales y académicos (VILLALOBOS et al., 2016). En términos étnicos, se encuentra evidencia que existe un efecto relacionado con la presencia de minorías étnicas en el establecimiento en términos académicos (BELLEI, 2013). Finalmente, también se ha indicado que la condición de migrante podría ser una variable relevante para entender la composición escolar, toda vez que los estudiantes migrantes se enfrentan actitudes de racismo y discriminación por parte de sus compañeros (TIJOUX, 2013) y que, además, estos tienden a distribuirse desigualmente en los distintos establecimientos (JOIKO; VÁSQUEZ, 2016).

Finalmente, también es posible reconocer la importancia de las variables académicas en la composición escolar. Partiendo de la idea de que intrínsecamente los estudiantes son diversos y distintos, se ha reconocido la existencia de variables académicas que generan, producen o amplifican posiciones de desventaja en el sistema escolar. En este sentido, se ha dado cuenta de que, por ejemplo, encontrarse en cursos con estudiantes de menores resultados académicos (VANDERHART, 2006), haber repetido en el sistema escolar (JIMERSON, 2001) o haber desarrollado trayectorias educativas inestables (GRIGG, 2012) repercute directamente en las oportunidades de aprendizaje y los resultados académicos. En Chile, se ha discutido intensamente sobre el efecto de la segregación académica entre establecimientos (BELLEI, 2013) y, más recientemente, sobre el efecto del agrupamiento por habilidad al interior de las escuelas (TREVIÑO et al., 2016).

\section{Antecedentes: el sistema escolar chileno}

El sistema escolar chileno se divide en dos grandes niveles: la educación básica o primaria, y la educación media o secundaria. La educación básica está compuesta por 8 grados $\left(1^{\circ}\right.$ básico a $8^{\circ}$ básico), mientras que la educación media está compuesta por $4\left(1^{\circ}\right.$ medio a $4^{\circ}$ medio). Este diseño supone el ingreso de los estudiantes a la educación básica a los 6 años de edad, a la educación media alrededor de los 14, y la finalización (teórica) de la escolaridad a los 17 o 18 años. El año 2018, habían 12021 establecimientos escolares, de los cuales el 65,83\% impartía educación básica, el 6,38\% educación media y el 27,79\% ambos niveles.

En cuanto al financiamiento escolar, desde los años ' 80 rige un sistema de voucher universal

que abarca tanto a los establecimientos públicos como particulares subvencionados del país, representando a cerca del $93 \%$ de la matrícula. Este sistema se basa en la entrega por parte del Estado 
de un subsidio por estudiante de acuerdo a la elección realizadas por las familias. De esta forma, el financiamiento educativo opera bajo una lógica de elección racional, en que la competencia por quedarse con el voucher de cada estudiante generará (teóricamente) una competencia entre las escuelas, buscando así generar las condiciones para la instalación de un mercado escolar (TREVIÑO et al., 2019).

El desarrollo extendido de este sistema, sumado a otras regulaciones escolares - como una política de rendición de cuentas con altas consecuencias, el sistema de admisión escolar o las políticas de libre elección - y a otras políticas nacionales — como la segregación residencial- han generado dos efectos relacionados con la composición escolar. En primer lugar, ha potenciado la provisión privada educativa, con la consecuente disminución de la oferta pública y diferenciación de la composición escolar. Para el año 2018, sólo un 43,9\% de la oferta educativa era pública, lo que sugiere una trayectoria bastante similar al caso brasileño en las últimas décadas (FALABELLA et al., 2019). En segundo término, el sistema escolar chileno presenta altos niveles de segregación escolar, produciendo una composición escolar diferenciada, especialmente (aunque no únicamente) en términos socioeconómicos (VALENZUELA et al., 2014). De esta forma, el sistema escolar chileno, al igual que el sistema escolar brasileño, puede caracterizarse como un sistema que posee niveles estructurales de segregación educativa (DA COSTA; BARTHOLO, 2014; ALVES et al., 2014).

\section{Metodología}

Datos

La investigación se basa en un análisis cuantitativo de distintas fuentes secundarias. Específicamente, se utiliza información del Sistema de Información General de Educación (SIGE) del Ministerio de Educación de Chile, e información proveniente del Sistema Integral de Mejoramiento de la Calidad Educativa (SIMCE) que aplica test estandarizados (de carácter censal) para estudiantes de $4^{\circ}$ y $8^{\circ}$ básico, y $2^{\circ}$ medio y que, además, recolecta información sobre los padres y estudiantes del sistema educativo. En ambos casos, la información utilizada es del año 2015.

El universo de estudio se compone de todos los establecimientos de educación diurna que dictan Enseñanza Básica (primaria) y/o Enseñanza Media (secundaria) con más de 20 estudiantes de matrícula total y que estuvieran en funcionamiento, excluyendo los establecimientos que entregaban exclusivamente educación especial y para adultos. En total, el universo corresponde a 7.099 establecimientos.

\section{Variables}

Para analizar la composición de los establecimientos se utilizaron 11 variables. La Tabla 1 detalla las variables utilizadas, la dimensión a la que corresponde, la forma de cálculo, su fuente de información y la naturaleza de la variable. Como se puede observar, se utilizaron variables de dos naturalezas distintas: variables dicotómicas y variables continuas. Como el objetivo de la investigación es analizar la composición o distribución de los estudiantes, en el caso de las variables dicotómicas se utilizó la proporción como medida de dispersión. En el caso de las variables continuas, se utilizó la desviación estándar como medida de análisis de la composición del establecimiento (las que, en el caso de calcularse para más de un nivel educativo, se promediaron). 
Tabla 1. Dimensiones, variables, forma de cálculo, naturaleza original de las variables y fuentes de información utilizadas.

\begin{tabular}{|c|c|c|c|c|}
\hline Dimensión & Variable & Forma de cálculo & $\begin{array}{c}\text { Naturaleza } \\
\text { original }\end{array}$ & $\begin{array}{l}\text { Fuente de } \\
\text { información }\end{array}$ \\
\hline \multirow{3}{*}{$\begin{array}{l}\text { Variables de } \\
\text { Composición } \\
\text { Cultural }\end{array}$} & Sexo del estudiante & Porcentaje de mujeres & Dicotómica & SIGE \\
\hline & $\begin{array}{l}\text { Origen étnico del } \\
\text { estudiante }\end{array}$ & $\begin{array}{c}\text { Porcentaje de estudiantes } \\
\text { pertenecientes a grupo étnico }\end{array}$ & Dicotómica & SIGE \\
\hline & $\begin{array}{l}\text { Nacionalidad del } \\
\text { estudiante }\end{array}$ & $\begin{array}{l}\text { Porcentaje de estudiantes } \\
\text { extranjeros }\end{array}$ & Dicotómica & SIGE \\
\hline \multirow{4}{*}{$\begin{array}{l}\text { Variables de } \\
\text { Composición } \\
\text { Académica }\end{array}$} & $\begin{array}{l}\text { Puntaje SIMCE } \\
\text { Lectura }\end{array}$ & $\begin{array}{l}\text { Promedio simple de desviación } \\
\text { estándar de puntaje en } 4^{\text {to }}, 8^{\text {vo }} \\
\text { básico y } 2^{\text {do }} \text { medio }\end{array}$ & Continua & SIMCE \\
\hline & $\begin{array}{l}\text { Puntaje SIMCE } \\
\text { Matemáticas }\end{array}$ & $\begin{array}{l}\text { Promedio simple de desviación } \\
\text { estándar de puntaje en } 4^{\text {to }}, 8^{\text {vo }} \\
\text { básico y } 2^{\text {do }} \text { medio }\end{array}$ & Continua & SIMCE \\
\hline & $\begin{array}{l}\text { Promedio General } \\
\text { de Notas }\end{array}$ & $\begin{array}{c}\text { Promedio simple de desviación } \\
\text { estándar de notas de cada nivel } \\
\text { del establecimiento }\end{array}$ & Continua & SIGE \\
\hline & $\begin{array}{l}\text { Condición de } \\
\text { alumno integrado }\end{array}$ & $\begin{array}{c}\text { Porcentaje de estudiantes con } \\
\text { necesidades educativas especiales }\end{array}$ & Dicotómica & SIGE \\
\hline \multirow{4}{*}{$\begin{array}{l}\text { Variables de } \\
\text { Composición } \\
\text { Socioeconómica }\end{array}$} & $\begin{array}{l}\text { Nivel educacional de } \\
\text { madre del estudiante }\end{array}$ & $\begin{array}{l}\text { Promedio simple de desviación } \\
\text { estándar de niveles educacionales } \\
\text { en } 4^{\text {to }}, 8^{\text {vo }} \text { básico y } 2^{\text {do }} \text { medio }\end{array}$ & Continua & SIMCE \\
\hline & $\begin{array}{l}\text { Nivel educacional de } \\
\text { padre del estudiante }\end{array}$ & $\begin{array}{l}\text { Promedio simple de desviación } \\
\text { estándar de niveles educacionales } \\
\text { en } 4^{\text {to }}, 8^{\text {vo }} \text { básico y } 2^{\text {do }} \text { medio }\end{array}$ & Continua & SIMCE \\
\hline & $\begin{array}{l}\text { Ingresos del hogar } \\
\text { per cápita }\end{array}$ & $\begin{array}{l}\text { Promedio simple de desviación } \\
\text { estándar de ingresos en } 4^{\text {to }}, 8^{\text {vo }} \\
\text { básico y } 2^{\text {do }} \text { medio }\end{array}$ & Continua & SIMCE \\
\hline & $\begin{array}{l}\text { Número de libros en } \\
\text { el hogar }\end{array}$ & $\begin{array}{l}\text { Porcentaje de estudiantes con más } \\
\text { de } 10 \text { libros en el hogar }\end{array}$ & Dicotómica & SIMCE \\
\hline
\end{tabular}

Análisis

El análisis se compone de dos pasos fundamentales. En primer lugar, y para agrupar a los establecimientos según el grado de homogeneidad o heterogeneidad en cada una de estas variables, se utilizó la técnica de cortes naturales, también conocido como método de optimización de Jenks (1967). Este método posee dos particularidades relevantes para este estudio. Por una parte, genera categorías sin requerir de otras variables adicionales para la construcción de los grupos, lo que lo diferencia de métodos como el análisis 
factorial o el análisis de clases latentes (VIVANCO, 1999). Por otra parte, y a diferencia de técnicas basadas en la distribución uniforme de categorías, como la construcción de quintiles o deciles, este método genera categorías a partir de la minimización de la varianza al interior de los grupos y la maximización de la varianza entre los grupos (JENKS, 1967, p. 186-190), lo que permite la obtención de grupos lo más homogéneos internamente (BALCERZAK, 2015).

Considerando la naturaleza de las variables, se decidió construir tres o cuatro grupos de establecimientos. Las variables dicotómicas fueron clasificadas en tres categorías: i) establecimientos homogéneos y concentrados en la categoría de referencia; ${ }^{1}$ ii) establecimientos homogéneos y concentradas en la categoría distinta a la de referencia; y iii) establecimientos heterogéneos. Por otro lado, las variables continuas fueron clasificadas en cuatro categorías: i) establecimientos heterogéneos; ii) establecimientos homogéneos y concentrados en la distribución alta de la categoría de referencia; iii) establecimientos homogéneos y concentrados en la distribución media de la categoría de referencia; y iv) establecimientos homogéneos y concentrados en la distribución baja de la categoría de referencia. La Tabla 2 detalla los puntos de corte establecidos para cada variable. Los puntos de corte se establecieron según la métrica de cada variable. Así, por ejemplo, en el caso del sexo del estudiante, el grupo heterogéneo considera a los establecimientos que tienen entre $40 \%$ y $73 \%$ de mujeres. Por otra parte, en el caso del puntaje SIMCE de Lectura, los establecimientos del grupo diverso son aquellos que tienen entre 14,15 y 81,31 desviaciones estándar promedio en esta prueba.

Tabla 2. Puntos de corte según método de Jenks para cada variable.

\begin{tabular}{|c|c|c|c|c|c|}
\hline \multirow[b]{2}{*}{ Dimensión } & \multirow[b]{2}{*}{ Variable } & \multirow[b]{2}{*}{ Heterogéneos } & \multicolumn{3}{|c|}{ Homogéneos } \\
\hline & & & $\begin{array}{l}\text { Concentrados } \\
\text { en distribución } \\
\text { baja de la } \\
\text { categoría de } \\
\text { referencia }\end{array}$ & $\begin{array}{l}\text { Concentrados } \\
\text { en distribución } \\
\text { media de la } \\
\text { categoría de } \\
\text { referencia }\end{array}$ & $\begin{array}{l}\text { Concentrados } \\
\text { en distribución } \\
\text { alta de la } \\
\text { categoría de } \\
\text { referencia }\end{array}$ \\
\hline \multirow{3}{*}{$\begin{array}{l}\text { Variables de } \\
\text { Composición } \\
\text { Cultural }\end{array}$} & Sexo del estudiante & $0,40-0,73$ & $0,00-0,39$ & & $0,74-1,00$ \\
\hline & Origen étnico del estudiante & $0,17-0,52$ & $0,00-0,16$ & & $0,53-1,00$ \\
\hline & Nacionalidad del estudiante & $0,07-0,24$ & $0,00-0,06$ & & $0,25-0,73$ \\
\hline \multirow{4}{*}{$\begin{array}{l}\text { Variables de } \\
\text { Composición } \\
\text { Académica }\end{array}$} & Puntaje SIMCE Lectura & $14,15-81,31$ & & $1,89-40,15$ & \\
\hline & Puntaje SIMCE Matemáticas & $39,47-76,05$ & & $3,02-39,46$ & \\
\hline & Promedio General de Notas & $0,53-1,08$ & & $0,18-0,53$ & \\
\hline & Condición de alumno integrado & $0,12-0,30$ & $0,00-0,11$ & & $0,31-0,92$ \\
\hline \multirow{4}{*}{$\begin{array}{l}\text { Variables de } \\
\text { Composición } \\
\text { Socio-económica }\end{array}$} & $\begin{array}{l}\text { Nivel educacional de madre } \\
\text { del estudiante }\end{array}$ & $2,88-6,57$ & & $0-2,88$ & \\
\hline & $\begin{array}{l}\text { Nivel educacional de padre } \\
\text { del estudiante }\end{array}$ & $2,94-6,19$ & & $0-2,94$ & \\
\hline & Ingresos del hogar per cápita & $2,13-5,96$ & & $0-2,13$ & \\
\hline & Número de libros en el hogar & $0,43-0,68$ & $0,00-0,42$ & & $0,69-1,00$ \\
\hline
\end{tabular}

Niveles educacional e ingresos son variables ordinales. En el caso de los niveles educacionales, existían 10 alternativas, desde sin educación formal (1) hasta postgrado (10). En el caso de ingresos del hogar, existieron 12 tramos, que van desde menos de \$200.000 (1) hasta más de $\$ 2.000 .000$ (12).

En segundo término, y considerando los resultados de este proceso de categorización, se utilizó la técnica de Análisis de Correspondencias Múltiples (ACM) para analizar las relaciones entre las distintas 
variables. El Análisis de Correspondencias Múltiples (ACM), permite "representar gráficamente la estructura de relaciones de dos o más variables mediante mapas de posicionamiento" (VIVANCO, 1999, p. 191) lo que, en nuestro caso, permitirá analizar la composición escolar considerando, al mismo tiempo, las dimensiones socioeconómicas, académicas y socioculturales. De esta forma, el análisis de ACM busca ver las relaciones entre las variables, más que entre los casos, como se realiza, por ejemplo, en el análisis de clusters, siendo especialmente útil para el estudio de la interdependencia de datos no continuos o categóricos (HAIR et al., 1995).

Dos modelos se probaron con esta técnica. El primero incorporó las 11 variables de composición del alumnado generadas. Este modelo proporcionó una varianza explicada de 61,6\% que si bien es alta (sobre $60 \%$ ), cuatro de las variables no se encontraban aportando información explicativa. El segundo modelo ACM (y final) se compuso, entonces, de 7 variables: i) origen étnico del estudiante; ii) SIMCE matemáticas; iii) promedio general de notas; iv) condición de estudiante integrado; v) ingresos por hogar; vi) educación de la madre; y vii) libros en el hogar. Al considerar sólo estas 7 variables, la varianza explicada por el modelo aumentó a un 77,3\%.

Adicionalmente, el ACM permite incorporar variables independientes para estudiar la correlación entre estas y el grupo de variables estudiadas. En nuestro caso, se incorporaron cuatro variables adicionales: la dependencia educacional del establecimiento (público, particular subvencionado o particular pagado); ii) el nivel de enseñanza impartido (educación básica, educación media o educación básica y media); iii) el tamaño del establecimiento (pequeños, medianos y grandes) ${ }^{2}$ y condición geográfica (rural o urbano).

El ACM permite un análisis de los datos mediante representaciones gráficas. Estas representaciones permiten identificar asociaciones entre las dimensiones consideradas a partir de la proximidad o distancia entre ellas (GATRELL et al., 2004). Así, las categorías de las variables posicionadas de manera cercana o próxima en el plano indican una alta asociación y, por tanto, la posibilidad de agruparlas (HAIR et al., 1995). Adicionalmente, las categorías cercanas al origen indican las categorías que estarían distribuidas de forma más aleatoria y, en consecuencia, no ayudarían a clasificar los establecimientos.

\section{Resultados}

En primer lugar, es interesante dar cuenta del nivel de homogeneidad y heterogeneidad del sistema escolar según el conjunto de variables estudiadas. Para ello, la Tabla 3 presenta la distribución porcentual de los establecimientos según la composición escolar de su estudiantado en las 11 variables de interés.

Tres principales resultados pueden desprenderse de la Tabla 3. En primer lugar, el sistema escolar chileno presenta diferencias importantes en el nivel de heterogeneidad de sus escuelas según las distintas variables analizadas. Así, en algunos casos, la cantidad de establecimientos heterogéneos es muy baja (como por ejemplo, respecto de la nacionalidad, el origen étnico del estudiante o los ingresos del hogar) mientras que en otros casos el porcentaje de establecimientos caracterizados como heterogéneos es bastante más alta (como ocurre respecto de la composición de género, la composición académica según el SIMCE o la composición socioeconómica según el nivel de escolaridad del padre o la madre). Esto estaría indicando que la posibilidad de que estudiantes de diversas culturas (distintas nacionalidades o etnias) se encuentren en un mismo establecimiento es menos frecuente que, por ejemplo, el encuentro de estudiantes con diverso rendimiento en pruebas estandarizadas. Probablemente, factores geográficos (como en el caso de la etnia), factores asociados al sistema de elección y selección escolar (en el caso de los estudiantes migrantes) o factores asociados a las barreras de entrada de los establecimientos (en el caso de los ingresos del hogar) podrían estar explicando los niveles de homogeneidad en estas variables. 
Tabla 3. Distribución porcentual de establecimientos según nivel de homogeneidad o heterogeneidad en su composición escolar $(n=7099)$.

\begin{tabular}{|c|c|c|c|c|c|}
\hline \multirow[b]{2}{*}{ Dimensión } & \multirow[b]{2}{*}{ Variable } & \multirow[b]{2}{*}{ Heterogéneos } & \multicolumn{3}{|c|}{ Homogéneos } \\
\hline & & & $\begin{array}{l}\text { Concentrados } \\
\text { en distribución } \\
\text { baja de la } \\
\text { categoría de } \\
\text { referencia }\end{array}$ & $\begin{array}{l}\text { Concentrados } \\
\text { en distribución } \\
\text { media de la } \\
\text { categoría de } \\
\text { referencia }\end{array}$ & $\begin{array}{c}\text { Concentrados } \\
\text { en distribución } \\
\text { alta de la } \\
\text { categoría de } \\
\text { referencia }\end{array}$ \\
\hline \multirow{3}{*}{$\begin{array}{l}\text { Variables de } \\
\text { Composición } \\
\text { Cultural }\end{array}$} & $\begin{array}{c}\text { Sexo del } \\
\text { estudiante }\end{array}$ & $85,12 \%$ & $12,30 \%$ & & $2,68 \%$ \\
\hline & $\begin{array}{l}\text { Origen étnico del } \\
\text { estudiante }\end{array}$ & $12,29 \%$ & $81,61 \%$ & & $6,10 \%$ \\
\hline & $\begin{array}{l}\text { Nacionalidad del } \\
\text { estudiante }\end{array}$ & $5,10 \%$ & $94,09 \%$ & & $0,91 \%$ \\
\hline \multirow{4}{*}{$\begin{array}{l}\text { Variables de } \\
\text { Composición } \\
\text { Académica }\end{array}$} & $\begin{array}{c}\text { Puntaje SIMCE } \\
\text { Lectura }\end{array}$ & $76,20 \%$ & $9,00 \%$ & $8,00 \%$ & $6,80 \%$ \\
\hline & $\begin{array}{c}\text { Puntaje SIMCE } \\
\text { Matemáticas }\end{array}$ & $58,7 \%$ & $20,3 \%$ & $13,06 \%$ & $8,04 \%$ \\
\hline & $\begin{array}{c}\text { Promedio General } \\
\text { de Notas }\end{array}$ & $44,61 \%$ & $5,99 \%$ & $25,26 \%$ & $24,34 \%$ \\
\hline & $\begin{array}{l}\text { Condición de } \\
\text { alumno integrado }\end{array}$ & $42,40 \%$ & $41,27 \%$ & & $16,43 \%$ \\
\hline \multirow{4}{*}{$\begin{array}{l}\text { Variables de } \\
\text { Composición } \\
\text { Socio-económica }\end{array}$} & $\begin{array}{c}\text { Nivel educacional } \\
\text { de madre del } \\
\text { estudiante }\end{array}$ & $60,13 \%$ & $9,85 \%$ & $15,37 \%$ & $14,75 \%$ \\
\hline & $\begin{array}{c}\text { Nivel educacional } \\
\text { de padre del } \\
\text { estudiante }\end{array}$ & $61,50 \%$ & $12,32 \%$ & $13,68 \%$ & $12,6 \%$ \\
\hline & $\begin{array}{l}\text { Ingresos del hogar } \\
\text { per cápita }\end{array}$ & $23,85 \%$ & $61,15 \%$ & $14,16 \%$ & $1,04 \%$ \\
\hline & $\begin{array}{l}\text { Número de libros } \\
\text { en el hogar }\end{array}$ & $44,33 \%$ & $29,30 \%$ & & $26,47 \%$ \\
\hline
\end{tabular}

En segundo lugar, los establecimientos que son homogéneos en su composición tienden a agrupar principalmente a estudiantes que se encuentran en la parte baja de la distribución, a lo menos en cuatro variables: i) origen étnico; ii) nacionalidad; iii) condición de alumno integrado; y iv) ingresos del hogar. Esto implica que, en general, los estudiantes de alguna etnia, migrantes, con necesidades educativas especiales y de bajos ingresos en general tienden a estar concentrados en los establecimientos educativos del sistema escolar chileno con mayor regularidad que aquellos estudiantes que no poseen estas características. Esto podría estar indicando que este conjunto de variables podría estar relacionándose para agrupar, en un mismo grupo de establecimientos, a estudiantes que poseen estas características.

Finalmente, llama la atención el alto porcentaje de establecimientos que, en dos variables, tienden a concentrar de manera homogénea a estudiantes que se encuentran en la parte alta de la distribución de las 
variables, como ocurre con el caso del promedio de notas y el número de libros existentes en el hogar. Esta concentración estaría mostrando que, en estos casos, existe una homogenización "por arriba", que tiende a generar que parte importante de los establecimientos del país concentre a estudiantes que tienen alto número de libros y promedios de notas altos, lo que estaría reflejando la importancia del capital cultural y las credenciales académicas en el proceso de homogenización en la composición escolar.

Ahora bien, para observar la relación entre las variables estudiadas, el Gráfico 1 presenta el resultado del Análisis de Correspondencias Múltiples (ACM). El gráfico se presenta en dos ejes que, en conjunto, explican más del 70\% del total de la varianza del modelo (el eje vertical explica un 49,14\% de la variabilidad de los datos y el eje horizontal explica un $28,14 \%$ de la varianza del modelo). Cada punto del gráfico representa una variable de la composición escolar y los números indican la categorización de los establecimientos según el grado de homogeneidad o heterogeneidad. Por otra parte, las variables independientes están representadas por los triángulos, permitiendo así visualizar la relación entre estas variables y las distintas medidas de composición.

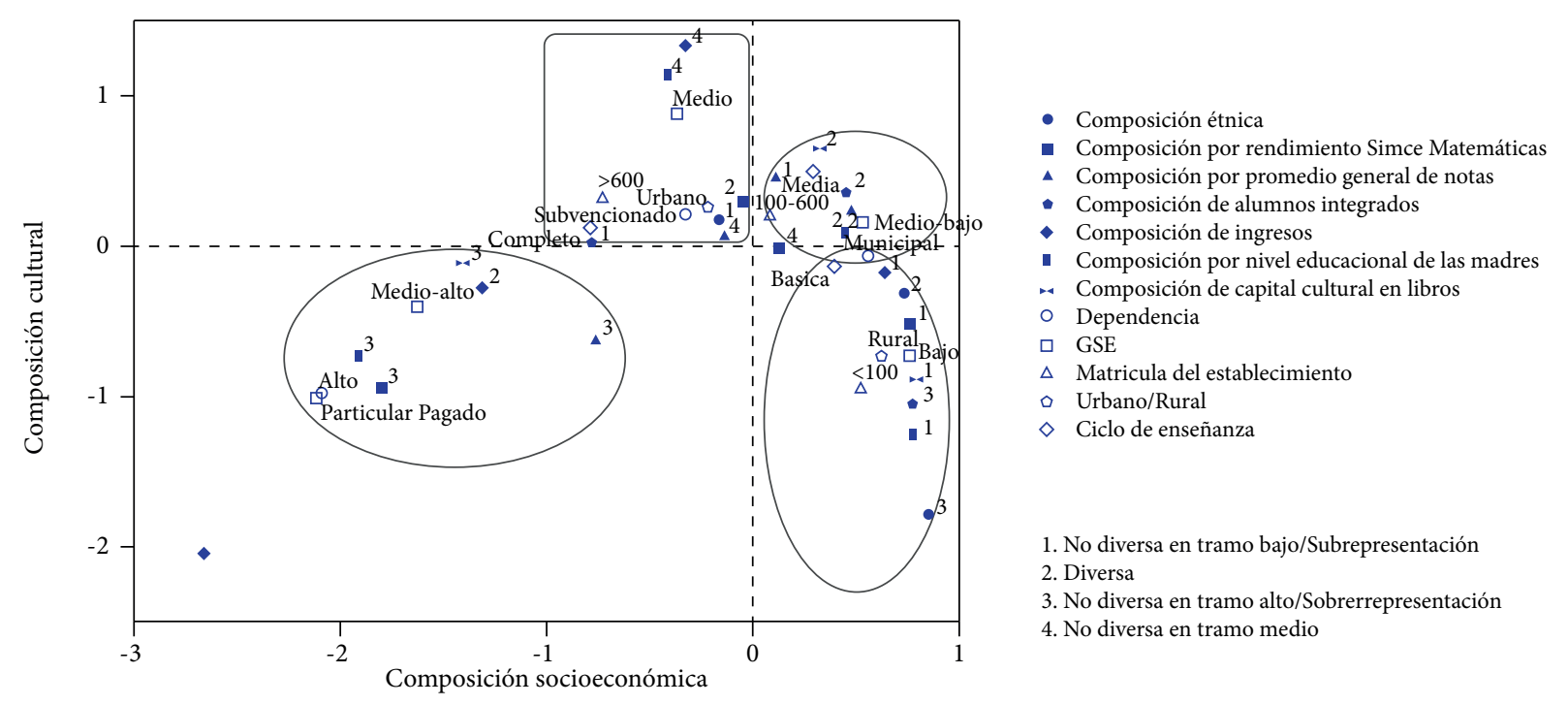

Figura 1. Análisis de Correspondencias Múltiples para características composicionales del alumnado de establecimientos educacionales chilenos.

Como se puede observar, existen cuatro grupos de establecimientos según su composición cultural, académica y socioeconómica. Un primer grupo, ubicado en el cuadrante inferior izquierdo del gráfico representa a establecimientos homogéneos en términos académicos y donde se encuentran estudiantes con altos puntajes en la prueba SIMCE, altos promedio de notas y bajo porcentaje de estudiantes con necesidades educativas especiales. Adicionalmente, estos establecimientos también tienden a ser homogéneos respecto del capital cultural, componiéndose por estudiantes que tienen alta cantidad de libros en el hogar y altos niveles de educación de sus madres (universitaria o más). Adicionalmente, estas características tienden a presentarse en los establecimientos particulares pagados. En síntesis, este primer grupo correspondería a los establecimientos de élite, caracterizados por tener bajos niveles de diversidad socioeconómica y académica y por concentrarse especialmente en establecimientos privados, en línea con lo indicado por la literatura disponible sobre este tópico (AGUILAR, 2011; MOYA; HERNÁNDEZ, 2014; BELLEI et al., 2020).

Un segundo grupo identificado se concentra en el cuadrante inferior derecho del plano. Una característica en común de este grupo con las escuelas de élite corresponde al alto nivel de homogenización 
de la composición escolar aunque, en este caso, esta homogenización está asociada a diversas formas de vulnerabilidad. De esta manera, este grupo incluye a estudiantes con resultados bajos en las pruebas estandarizadas y una alta proporción de estudiantes con necesidades educativas especiales. Además, en general presenta una composición homogénea en términos socioeconómicos, con ingresos bajos (menores a $\$ 400.000$, es decir, cerca de 600 USS), bajos niveles educacionales de las madres (en general, educación básica o menos) y baja presencia de libros en el hogar, mostrando así la interacción entre distintas formas de vulnerabilidad (HALL, 1980). Esta composición escolar se asocia a establecimientos con características particulares: establecimientos municipales, que se ubican en zonas rurales, que entregan educación básica y que son pequeños, lo que podría explicar la diversidad étnica que presentan.

A diferencia de estos dos grupos de establecimientos, existe un tercer grupo, ubicado en el cuadrante superior izquierdo, que presenta composiciones escolares que difieren según las variables de estudio. Por una parte, son establecimientos homogéneos en términos socioeconómicos (educación de la madre e ingresos) agrupando a estudiantes cuyas familias tienen ingresos medios y principalmente a madres con educación media completa. Adicionalmente, estos establecimientos también son homogéneos en términos de la proporción de estudiantes de etnia que integran. Sin embargo, al mismo tiempo, presenta niveles de heterogeneidad en términos académicos, especialmente respecto de la prueba SIMCE. A diferencia del grupo anterior, estos establecimientos son homogéneos étnicamente, y tienden a relacionarse con establecimientos de dependencia particular-subvencionada, urbanos y que entregan educación completa, lo que podría estar relacionado con lo que la literatura ha reconocido como "establecimientos de la clase media" (CANALES et al., 2016).

Finalmente, existe un cuarto grupo de establecimientos, que se visualiza por variables ubicadas en el cuadrante superior derecho. A diferencia de los otros tres, este grupo se caracteriza por contener altos niveles de heterogeneidad en distintas variables de la composición escolar. Así, muestran una composición heterogénea respecto de variables socioeconómicas, como el capital cultural y el nivel educacional de la madre, pero también respecto de variables socioeconómicas, como la proporción de estudiantes con necesidades educativas especiales, el rendimiento escolar y los resultados en la prueba estandarizada de matemáticas (SIMCE). Asimismo, este grupo se presenta especialmente en establecimientos municipales, de educación media y en establecimiento de tamaño mediano, esto es, entre 100 y 600 estudiantes.

\section{Conclusiones}

El objetivo del estudio fue analizar la composición escolar de los establecimientos educativos chilenos desde una perspectiva multidimensional. En general, los resultados muestran que las dimensiones académicas, socioeconómicas y socioculturales de la composición escolar tienden a presentarse de manera conjunta en los establecimientos, lo que implica que los establecimientos con altos (o bajos) niveles de homogeneidad tienden concentran a estudiantes de similares características en varias de las variables analizadas. Adicionalmente, los resultados muestran que existe una marcada diferenciación en tres de los cuatro grupos analizados, que tienden a concentrar a estudiantes de la élite (grupo 1), a estudiantes vulnerables (grupo 2) y a estudiantes de clase media (grupo 3). A pesar de esto, existe, no obstante, un grupo de establecimientos que presenta mayores niveles de diversidad, especialmente en términos socioeconómicos y académicos (grupo 4).

Este conjunto de resultado permite potenciar análisis y discusiones respecto de las políticas públicas y la investigación educativa y la discusión sobre inclusión educativa, integración social y justicia educacional. 
Respecto de las políticas educativas, los resultados complementan y profundizan discusiones en a lo menos dos ámbitos.

Por un lado, parte importante de la discusión pública en la última década se ha centrado en la segregación educativa que se produce entre establecimientos, que ha mostrado que, en general, el sistema educacional chileno produce bajos niveles de interacción entre estudiantes de características distintas (BELLEI, 2013). Esta investigación corrobora estos datos, pero muestra que otras características (como la nacionalidad, la etnia o el capital cultural) también se encuentran, en general, concentradas y distribuidas bastante homogéneamente en el sistema escolar, lo que podría impulsar una nueva generación de políticas educativas focalizadas a promover mayores niveles de integración en estas características considerando, de esta manera, múltiples variables de la composición educativa. Un ejemplo claro de esto podría ser la forma de asignación financiera que, hoy en día, se desarrolla casi exclusivamente a través de variables socioeconómicas.

Por otra parte, los resultados muestran que, además de multidimensional, los procesos de homogenización se producen en la parte alta, baja y media de la distribución de las distintas variables de la composición escolar. Esto implica que el sistema escolar está configurado, en distintos niveles, para promover procesos de separación, lo que puede entenderse como un obstáculo para la cohesión social y un aliciente para el desarrollo de prejuicios y conflictos sociales (VILLALOBOS; VALENZUELA, 2012). Esto es relevante para las políticas públicas, pues implica que todos los grupos del sistema deben verse inquiridos para mostrar mayores niveles de mixtura y diversidad social. Hasta la fecha, los cambios generados por la política educativa se han focalizado especialmente en los grupos vulnerables y, en menor medida, en los grupos medios, sin generar mayores políticas de integración de los grupos altos o de la élite, los que, además, son los que más diferenciados se encuentran de otros grupos (VALENZUELA et al., 2013) y los que construyen mayores barreras sociales de diferenciación (MOYA; HERNÁNDEZ, 2014) aún en espacios públicos (QUARESMA, 2015).

Por parte de la investigación educativa, también aparecen importantes desafíos. En primer término, es necesario entender de manera más profunda el proceso de elección y selección escolar desde la perspectiva multidimensional. Hasta la fecha, la literatura sobre elección (HERNÁNDEZ; RACZYNSKI, 2015; KOSUNEN; CARRASCO, 2016) y selección escolar (CARRASCO et al., 2017; CONTRERAS et al. 2010) se ha focalizado sobretodo en entender el rol de las variables socioeconómicas y académicas. Entender cómo otras variables de la composición escolar influyen en estos procesos (sobre todo en el marco de construcción de un nuevo sistema de admisión) aparece como un objetivo de investigación de corto y mediano plazo. En este sentido, parece especialmente relevante profundizar en la comprensión del rol que las barreras geográficas y la configuración de las grandes ciudades puede tener en los procesos de segregación y composición escolar, aspecto abordado de manera incipiente en estudios tanto en Brasil como Chile (ELACQUA, 2012; DA COSTA; BARTHOLO, 2014)

En segundo término, es necesario potenciar investigaciones que potencien el estudio cualitativo de la composición escolar y la forma en como estas se desenvuelven en el espacio escolar. Entender los procesos de etiquetamiento y categorización - fundamentalmente subjetivos- que se producen en las escuelas permitirá entender de mejor manera la forma en cómo la composición escolar es vivenciada y los efectos que esto puede tener en la inclusión escolar. Hasta la fecha, la investigación existente se ha concentrado en entender este efecto en grupos focalizados de sujetos, como los estudiantes migrantes (TIJOUX, 2013), pero no ha profundizado mayormente en la forma en cómo las distintas variables de la composición escolar interactúan entre sí. De ahí la necesidad de profundizar en estudios etnográficos y/o estudios de percepciones que indaguen en estas perspectivas.

Finalmente, parece necesario profundizar en el conocimiento de las características y factores que potencian la heterogeneidad en los establecimientos. Como se pudo observar, existe un grupo de 
establecimientos que -contracorriente del conjunto del sistema- si presenta altos niveles de heterogeneidad en términos socioeconómicos, socioculturales y académicos. Por lo mismo, impulsar estudios de casos que permitan conocer en profundidad estos establecimientos, las características de su composición y los efectos que esta configuración heterogénea tiene, pueden ser un desafío interesante para próximas investigaciones.

\section{Contribuición de los autores}

Conceptualización de la investigación, Villalobos C; Revisión de la literatura, Villalobos C, Ramírez P, Infante I; Instrumentos de recolección y análisis de dados, Ramírez P, Infante I; Discusión, Villalobos C, Wyman I; Escritura, Villalobos C, Wyman I; Corrección de estilo, Villalobos C, Wyman I

\section{Notas}

1. Como se muestra en la Tabla 1, las categorías de referencia fueron el porcentaje de mujeres, porcentaje de estudiantes de origen étnico, porcentaje de estudiantes extranjeros, porcentaje de estudiantes con necesidades educativas especiales y porcentaje de estudiantes con más de 10 libros en el hogar.

2. Por establecimientos pequeños se consideraron aquellos menores a 100 alumnos. Los establecimientos medianos fueron aquellos con una matrícula de entre 101 y 600 alumnos. Los establecimientos grandes fueron aquellos con sobre 600 estudiantes.

\section{Referencias}

AGUILAR, O. Dinero, educación y moral: El cierre social de la elite tradicional chilena. In: JOIGNANT, A.; GÜELL, P. (eds.). Notables, tecnócratas y mandarines. Elementos de Sociología de las élites en Chile (19902010). Santiago: Ediciones Universidad Diego Portales, 2011, p. 185-202.

ALVES, F. et al. Winners and losers of school choice: Evidence from Rio de Janeiro, Brazil and Santiago, Chile. International Journal of Educational Development, v. 41, p. 25-34, 2014. https://doi.org/10.1016/j. ijedudev.2014.12.004

ASCORRA, P. et al. Relación entre segregación y convivencia escolar en escuela públicas chilenas. Universitas Psychologica, v. 15, n. 1, p. 65-78, 2016. http://doi.org/10.11144/Javeriana.upsy15-1.rsce

BALCERZAK, A. Europe 2020 Strategy Implementation. Grouping the countries with the application of natural breaks method. 8th International Conference on Applied Economics Contemporary Issues in Economy: Market or Government? Polish Economic Society, Toruń, Poland, June 2015

BELLEI, C. El estudio de la segregación socioeconómica y académica de la educación chilena. Estudios Pedagógicos, v. 39, n. 1, p. 325-345, 2013. http://doi.org/10.4067/S0718-07052013000100019

BELLEI, C. Dificultades y resistencias de una reforma para dificultades y resistencias de una reforma. RASE - Revista de la Asociación de Sociología de la Educación, v. 9, n. 2, p. 232-247, 2016.

BELLEI, C.; ORELLANA, V.; CANALES, M. Elección de escuela en la clase alta chilena. Comunidad, identidad y cierre social. Archivos Analíticos de Políticas Educativas, v. 28, n. 5, p. 1-27, 2020 
BOURDIEU, P. The forms of capital. In: HALSEY, A. H. (ed.). Education: Culture, Economy, and Society, Oxford: Oxford Universitu Press, 1986, p. 46-58.

BRADLEY, R.; CORWYN, R. Socioeconomic status and child development. Annual Review of Psychology, v. 53, p. 371-399, 2002. https://doi.org/10.1146/annurev.psych.53.100901.135233

CALDAS, S.; BANKSTON, C. Effect of school population socioeconomic status on individual academic achievement. Journal of Educational Research, v. 90, n. 5, p. 269-277, 1997. https://doi.org/10.1080/00 220671.1997.10544583

CANALES, M.; BELLEI, C.; ORELLANA, V. ¿Por qué elegir una escuela particular-subvencionada? Sectores medios emergentes y elección de escuela en un sistema de mercado. Estudios Pedagógicos, v. 42, n. 3, p. 89-109, 2016. http://doi.org/10.4067/S0718-07052016000400005

CARRASCO, A.; GUTIÉRREZ, G.; FLORES, C. Failed regulations and school composition: Selective admission practices in Chilean primary schools. Journal of Education Policy, v. 32, n. 5, p. 642-672, 2017. https://doi.org/10.1080/02680939.2017.1312549

CONTRERAS, D.; SEPÚlVEDA, P.; BUSTOS, S. When schools are the ones that choose: The effects of screening in Chile. Social Science Quarterly, v. 91, n. 5, p. 1349-1368, 2010.

COSTA, S. Desigualdades, interdependências e afrodescendentes na América Latina. Tempo Social, v. 24, n. 2, p. 123-145, 2012.

DA COSTA, M.; BARTHOLO, T. L. Padrões de segregação escolar no Brasil: Um estudo comparativo entre capitais do país. Educação \& Sociedade, v. 35, n. 129, p. 1183-1203, 2014. https://doi.org/10.1590/ ES0101-73302014144444

DUK, C.; LOREN, C. Flexibilización del currículum para atender la diversidad. Revista Latinoamericana de Inclusión Educativa, v. 4, n. 1, p. 187-210, 2010.

DUPRIEZ, V. Methods of Grouping Learners at School. Paris: UNESCO, 2010.

DURKHEIM, E. Educación y Sociología. Barcelona: Península, 1975.

ELACQUA, G. The impact of school choice and public policy on segregation: Evidence from Chile. International Journal of Educational Development, v. 32, n. 3, p. 444-453, 2012. https://doi.org/10.1016/j. ijedudev.2011.08.003

FALABELLA, A; PIRES, D.; PERONI, V. Los modos de privatización en Brasil y Chile y las implicancias para la democratización de la educación pública. RIAEE - Revista Ibero-Americana de Estudos em Educação, v. 14, n. 3, p. 1813-1828, 2019.

FREEMAN, K.; STEIDL, C. Distribution, composition and exclusion: How school segregation impacts racist disciplinary patterns. Race and Social Problems, v. 8, n. 2, p. 171-185, 2016. https://doi.org/10.1007/ s12552-016-9174-9

GATRELL, A.; POPAY, J.; THOMAS, C. Mapping the determinants of health inequalities in social space: Can Bourdieu help us? Health \& Place, v. 10, n. 3, p. 245-257, 2004. https://doi.org/10.1016/j.healthplace.2003.09.005

GRIGG, J. School enrollment changes and student achievement growth. Sociology of Education, v. 85, n. 4, p. 388-404, 2012. https://doi.org/10.1177/0038040712441374 
HAIR, J. et al. Multivariate data analysis. Upper Saddle River: Prentice Hall., 1995.

HALL, S. Race, articulation and societies structured in dominance. In: UNESCO (ed.). Sociological Theories: Race and Colonialism. Paris: UNESCO, 1980. https://doi.org/10.1215/9781478002413-010

HANUSHEK, E.; WOESSMAN, L. The Economics of International Differences in Educational Achievement. National Bureau of Economic Research, 2010.

HERNÁNDEZ, M.; RACZYNSKI, D. Elección de escuela en Chile: De las dinámicas de distinción y exclusión a la segregación socioeconómica del sistema escolar. Estudios Pedagógicos, v. 2, p. 127-141, 2015. http:// dx.doi.org/10.4067/S0718-07052015000200008

JANMAAT, J. G. Educational differentiation and inequalities in civic education. In: JAMMAAT, J. et al. (eds.). The Dynamics and Social Outcomes of Education Systems. London: Palgrave-MacMillan, 2013. https:// doi.org/10.1057/9781137025692_10

JANMAAT, J. G. Do ethnically mixed classrooms promote inclusive attitudes towards immigrants everywhere? A study among native adolescents in 14 countries. European Sociological Review, v. 30, n. 6, p. 810-822, 2014. https://doi.org/10.1093/esr/jcu075

JENKS, G. The data model concept in statistical mapping. International Yearbook of Cartography, v. 7, n. 1, p. 186-190, 1967.

JIMERSON, S. Meta-analysis of grade retention research: Implications for practice in the $21^{\text {st }}$ century. School Psychology Review, v. 30, n. 3, 2001.

JOIKO, S.; VÁSQUEZ, A. Acceso y elección escolar de familias migrantes en Chile: No tuve problemas porque la escuela es abierta, porque acepta muchas nacionalidades. Calidad en la Educación, n. 45, p. 132-173, 2016. http://doi.org/10.4067/S0718-45652016000200005

KELLY, S. Classroom discourse and the distribution of student engagement. Social Psychology of Education, v. 10, n. 3, p. 331-352, 2007. https://doi.org/10.1007/s11218-007-9024-0

KOSUNEN, S.; CARRASCO, A. Parental preferences in school choice: Comparing reputational hierarchies of schools in Chile and Finland. Compare, v. 46, n. 2, p. 172-193, 2016. https://doi.org/10.1080/03057925. 2013.861700

MCDONNELL, L.; TIMPANE, M.; BENJAMIN, R. Rediscovering the Democratic Pourpose of Education. Lawrance: University of Kansas Press, 2000.

MIZALA, A.; TORCHE, F. Bringing the schools back in: The stratification of educational achievement in the Chilean voucher system. International Journal of Educational Development, v. 32, n. 1, p. 132-144, 2012. https://doi.org/10.1016/j.ijedudev.2010.09.004

MOODY, J. Race, school integration, and friendship segregation in America. American Journal of Sociology, v. 107, n. 3, p. 679-716, 2001. https://doi.org/10.1086/338954

MOYA, E.; HERNÁNDEZ., J. El rol de los colegios de elite en la reproducción intergeneracional de la elite Chilena. Revista Austral de Ciencias Sociales, v. 1, n. 26, p. 59-82, 2014. https://doi.org/10.4206/rev.austral. cienc.soc.2014.n26-04 
OPDENAKKER, M-C.; VAN DAMME, J. Relationship between school composition and characteristics of school process and their effect on mathematics achievement. British Educational Research Journal, v. 27, n. 4, p. 407-432, 2001. https://doi.org/10.1080/01411920120071434

QUARESMA, M. L. Entre a entrega e a renúncia: excelência acadêmica em escolas públicas chilenas de alta performance. Educação e Pesquisa, v. 41, p. 1487-1502, 2015. https://doi.org/10.1590/ S1517-9702201508141701

RIEDEMANN, A. Alumnos inmigrantes en escuelas chilenas: Una mirada a las desigualdades del sistema educativo. In: VERA, A. (ed.). Malestar social y Desigualdades en Chile. Santiago: Ediciones Universidad Alberto Hurtado, 2017, p. 99-124.

SERRANO, S.; PONCE DE LEÓN, M.; RENGIFO, F. Historia de la educación en Chile (1810-2010). Santiago: Taurus, 2012.

SHAFIQ, M.; MYERS, J. Educational vouchers and social cohesion: a statistical analysis of student civic attitudes in Sweden, 1999-2009. American Journal of Education, v. 12, n. 1, p. 111-136, 2014. https://doi. org/10.1086/678115

TIJOUX, M. E. Niños(as) marcados por la inmigración peruana: estigma, sufrimientos, resistencias. Convergencia. Revista de Ciencias Sociales, v. 20, n. 61, p. 83-104, 2013.

TREVIÑO, E.; VALENZUELA, J. P.; VILLALOBOS, C. Within-school segregation in the Chilean school system: What factors explain it? How efficient is this practice for fostering student achievement and equity? Learning and Individual Differences, v. 51, p. 367-375, 2016. https://doi.org/10.1016/j.lindif.2016.08.021

TREVIÑO, E.; VALENZUELA, J. P.; VILLALOBOS, C. Segregación de estudiantes indígenas en el sistema escolar chileno. In: TREVIÑO, E. et al. (eds.). Educación Intercultural Bilingue en Chile. Experiencias, Pueblos y Territorios. Santiago: Ediciones UC, 2017

TREVIÑO, E. et al. Agrupamiento por habilidad académica en el sistema escolar. Nueva evidencia para comprender las desigualdades del sistema educativo chileno. Revista Mexicana de Investigación Educativa, v.23, n.73, p. 45-71, 2018.

TREVIÑO, E. et al. Financiamiento de la educación escolar en Chile: La reforma estructural pendiente. In: CARRASCO, A.; FLORES, L. (eds.) De la reforma a la transformación. Capacidades, innovaciones y regulación de la educación chilena. Santiago: Ediciones UC, 2019, p. 505-544.

VALENZUELA, J. P.; VILLALOBOS, C.; GÓMEZ, G. Segregación y polarización en el sistema escolar chileno. ¿Qué ha sucedido con los grupos medios? Documento de Referencia n. 03, ago. 2013.

VALENZUELA, J. P.; BELLEI, C.; DE LOS RÍOS, D. Socioeconomic school segregation in a market-oriented educational system. The case of Chile. Journal of Education Policy, v. 29, n. 2, p. 217-241, 2014. https://doi. org/10.1080/02680939.2013.806995

VALENZUELA, J. P.; MONTECINOS, C. Structural reforms and equity in Chilean schools. Oxford Research Encyclopedia of Education, 2017. https://doi.org/10.1093/acrefore/9780190264093.013.108

VANDERHART, P. G. Why do some schools group by ability? Some evidence from the NAEP. American Journal of Economics and Sociology, v. 65, n. 2, p. 435-462, 2006. https://doi.org/10.1111/j.1536-7150.2006.00458.x 
VERGER, A.; BONAL, X.; ZANCAJO, A. Recontextualización de políticas y (cuasi)mercados educativos. Un análisis de las dinámicas de demanda y oferta escolar en Chile. Aape-Epaa, v. 24, n. 27, p. 1-27, 2016. https://doi.org/10.14507/epaa.24.2098

VILLALOBOS, C. El campo educativo en Chile post-dictadura (1990-2013). Continuidad y ruptura en la implementación del neoliberalismo en educación. Democracia versus neoliberalismo. 25 años de neoliberalismo en Chile. Santiago: Fundación Rosa Luxemburgo/ICAL, 2016, p. 159-178.

VILLALOBOS, C.; VALENZUELA, J. P. Polarizacion y cohesion social del sistema escolar chileno. Revista de Análisis Económico, v. 27, n. 2, p. 145-172, 2012. http://doi.org/10.4067/S0718-88702012000200005

VILLALOBOS, C. et al. Composición de género en establecimientos escolares chilenos: ¿Afecta el rendimiento académico y el ambiente escolar? Estudios Pedagógicos, v. 42, n. 2, p. 379-394, 2016. http://doi.org/10.4067/ S0718-07052016000200022

VIVANCO, M. Análisis Estadístico Multivariable. Santiago: Editorial Universitaria, 1999.

WILLIAMSON, G. Institucionalización de la educación intercultural bilingüe en Chile: Notas y observaciones críticas perfiles educativos. Perfiles Educativos, v. 34, p. 126-147, 2012.

\section{Sobre los Autores}

Cristóbal Villalobos es doctor en Ciencias Sociales de la Universidad de Chile. Magister en Economía Aplicada de la Universidad Alberto Hurtado y Georgetown University. Sociólogo y Trabajador Social de la Pontificia Universidad Católica de Chile. Actualmente, es Investigador Asociado del Centro de Estudios de Políticas y Prácticas en Educación (CEPPE UC). Sus áreas de investigación son calidad y equidad educativa, educación cívica, educación superior y movimientos sociales.

Paz Ramírez es socióloga y minor en Economía de la Pontificia Universidad Católica de Chile.

Isabel Infante es socióloga de la Pontificia Universidad Católica de Chile. Actualmente, trabaja en el área de envejecimiento, territorio y comunidades.

Ignacio Wyman es sociólogo de la Pontificia Universidad Católica de Chile. Investigador Adjunto del Centro de Estudios de Políticas y Prácticas en Educación (CEPPE-UC). Ha participado en proyectos de investigación que abarcan políticas y prácticas de segregación en el sistema escolar chileno y al interior de las escuelas, estudios sobre diversidad e inclusión escolar, evaluación de programas y políticas educativas a nivel inicial y escolar, y estudios comparados en educación cívica.

Recibido: 06 Jun 2018

Aceptado: 07 Feb 2020 